\title{
Compact thermal model of a D2Pak case with convection
}

\author{
by M. Lis, B. Wiecek and K. Tomalczyk \\ Institute of Electronics, Technical University of Lodz, Poland
}

\begin{abstract}
The paper presents a compact thermal model of an electronic component enclosed in a D2Pak case. The model includes convection cooling and is compared to a linear model with isothermal boundary condition. The model presented is a static model.
\end{abstract}

\section{Introduction}

A compact thermal model is a simple network of about ten to twenty nodes. Thanks to the low number of nodes, the time required to compute the output variable - the junction temperature, is considerably reduced. The model consists of any finite numer of branches connecting the external (surface) and internal nodes.

The model considered up to now was a linear model, being only a rough approximation of reality. To build a complete model it is essential to include the heat transfer phenomena between the component and its environment, like convection and radiation. At the present stage of the study, the convection is considered.

To calculate the parameters of a compact thermal model, the temperature distribution in the investigated component must be simulated or measured. A thermal camera is an excellent tool to determine the external temperatures of the component without influencing its structure, which is very difficult to do using contact temperature measurement techniques. The thermal images provide information about the temperature of the characteristic points of the component, chosen to be the nodes of the compact thermal model. These temperature values are then used to calculate the junction temperature.

\section{Thermal model of the D2Pak component}

A finite element model of a D2Pak case power transistor together with its dedicated printed circuit board (PCB) was created using the ANSYS 5.7 simulation environment and based on the component parameters taken from the manufacturer's data sheets. Figure 1a shows the actual position of the active element (the junction) within the structure of the component. Figure $1 \mathrm{~b}$ is the general view of the modelled transistor placed on the PCB. Figure 1c presents the base structure for the compact thermal model. The seven nodes are: junction (in the middle), case, connections, paths, soldering pad, substrate and PCB. The nodes are joined together with the use of model parameters $R_{t h z}, a_{i}, q_{k}, R_{i, k}$. The additional node is introduced for the ambient temperature. This node is connected to the remaining part of the model by means of the convection coefficient $\alpha_{k}$. 


\section{http://dx.doi.org/10.21611/qirt.2004.072}

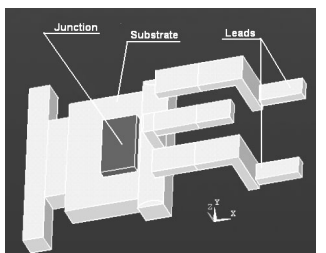

$1 a$

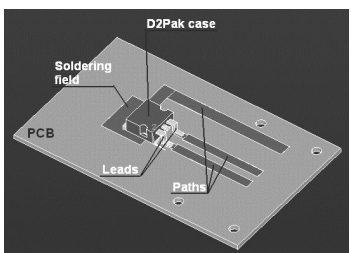

$1 b$

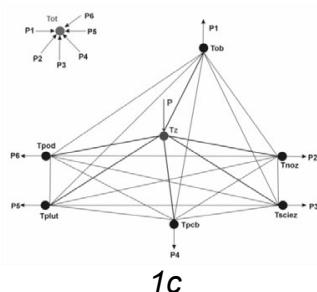

Fig. 1. Structure of the model

$T_{z}$ - junction, $T_{o b}$ - case, $T_{n o z}-$ connections, $T_{\text {sciez }}-$ paths, $T_{p c b}-P C B$,

$\mathrm{T}_{\text {plut }}$ - soldering pad, $\mathrm{T}_{\text {pod }}$ - substrate, $\mathrm{T}_{\text {otocz }}$ - ambient, $\mathrm{P}-$ power dissipated in the junction, $\mathrm{P}_{1} \ldots \mathrm{P}_{6}$ - power flowing towards the environment through the particular (1..6) node of the model.

\section{Static linear model}

The compact thermal model is based on two fundamental equations (1) and (3). The first equation (1) describes the dependency of the junction temperature on the dissipated power and on the temperatures of thermal contacts (external nodes) [2].

$T_{z}=P \cdot R_{t h z}+\sum_{i=1}^{n} T_{i} \cdot a_{i}$

where: $R_{\text {thz }}, a_{i}$ - model parameters, $P=\int_{v} P_{v} d v$ - power dissipated in the junction, $T_{i}(i=1, \ldots, n)$ - temperature at the particular node.

The $a_{i}$ coefficients, describing the influence of thermal contacts temperature on the junction temperature, must satisfy the constraint (2).

$\sum_{i=1}^{n} a_{i}=1$

Generally, the equation (1) is sufficient to describe the model, but different components having the same temperature distribution may have different heat flows through the external surfaces. To complete the model the second equation (3) is added. The equation (3) describes the total heat flow through the particular node.

$P_{k}=P \cdot q_{k}+\sum_{i=1}^{n} \frac{T_{i}}{R_{i, k}}$

where: $R_{i, k}, q_{k}$ - model parameters.

The $R_{i, k}$ coefficients determine how the heat flowing out of the node $k$ is influenced by the temperature at the remaining nodes. The $q_{k}$ coefficients, describing how much of the total power from the junction flows out through the particular node, must satisfy the similar constraint as the $a_{i}$ coefficients do (4).

$\sum_{k=1}^{n} q_{k}=1$ 
Thanks to the symmetry of the $\mathrm{R}_{\mathrm{i}, \mathrm{k}}$ parameters $\left(\frac{1}{R_{i, i}}=-\sum_{k=1, k \neq i}^{n} \frac{1}{R_{i, k}}\right)$, and to the constraints (2) and (4), the total number of $(n+1)^{2}$ model parameters reduces to the number of independent parameters (5):

$\frac{1}{2}(n-1) \cdot(n+4)+1$

where $n$ - total number of nodes (Fig.1).

Equations (1), (3) and constraints (2), (4) and $\left(\frac{1}{R_{i, i}}=-\sum_{k=1, k+i}^{n} \frac{1}{R_{i, k}}\right)$ are fundamental

for the creation of a compact thermal model with parameters $R_{t h z}, a_{i}, q_{k}, R_{i, k}$, obtained by thermal measurements or simulations. The model is linear and therefore the solutions for particular non-zero excitations $\left(P, T_{i}\right)$ can be superimposed to obtain the general solution. The model parameters $R_{\text {thz }}$, and $q_{k}$ are determined for all $T_{i}=0$ and $P \neq 0$, the $a_{i}$, and $R_{i, k}-$ for $P=0$ and each $T_{i} \neq 0, T_{k \neq i}=0$ respectively. In each of the above cases a finite element simulation is run to calculate the values of $T_{z}$ and $P_{\mathrm{i}}$.

\section{Static model with natural convection}

In the case considered for the model (laminar flow of the medium along the flat and cylindrical surfaces [3]), the convection coefficient $\alpha_{i}\left[\mathrm{~W} / \mathrm{m}^{2} \cdot \mathrm{K}\right]$ can be calculated via the following formulas [3] [1]:

- for the horizontal surface, hot side up

$\alpha_{i}=1.30 \cdot A_{i} \cdot\left(\frac{T_{i}-T_{o t}}{L_{i}}\right)^{\frac{1}{4}}$

- for the horizontal surface, hot side down

$\alpha_{i}=0.7 \cdot A_{i} \cdot\left(\frac{T_{i}-T_{o t}}{L_{i}}\right)^{\frac{1}{4}}$

where: $L_{i}-$ characteristic dimension of the surface $[\mathrm{m}]$.

The $A$ coefficient depends of the physical parameters of the environment: thermal conductivity, kinetic viscosity, gravity constant, volume expansion temperature coefficient. The formula to calculate the amount of power flowing out to the environment through a particular node is derived from the Newton's law (8) [4]:

$P_{k}=\alpha_{k} \cdot\left(T_{k}-T_{o t}\right) \cdot S_{k}$

The convection coefficient $\alpha_{\mathrm{ki}}$ is then substituted by the formula (6) or (7) to obtain the final equation (9):

$P_{k}=1.3 \cdot A_{k} \cdot\left(\frac{T_{k}-T_{o t}}{L_{k}}\right)^{\frac{1}{4}} \cdot\left(T_{k}-T_{o t}\right) \cdot S_{k}$

where: $S_{i}-$ the surface taking part in the heat transfer $\left[\mathrm{m}^{2}\right]$

Finally, combining (9) with (3) results in a new, non-linear system of equations (1014) describing the model with convection: 


$$
\begin{aligned}
& T_{z}=P \cdot R_{t h z}+\sum_{i=1}^{n} T_{i} \cdot a_{i} \\
& 1,3 \cdot A_{k} \cdot S_{k} \cdot\left(\frac{T_{k}-T_{o t}}{L_{k}}\right)^{\frac{1}{4}} \cdot\left(T_{k}-T_{o t}\right)=P \cdot q_{k}+\sum_{i=1}^{n} \frac{T_{i}}{R_{i, k}} \\
& \sum_{i=1}^{n} a_{i}=1 \\
& \sum_{k=1}^{n} q_{k}=1 \\
& \frac{1}{R_{i, i}}=-\sum_{k=1, k \neq i}^{n} \frac{1}{R_{i, k}}
\end{aligned}
$$

Introduction of non-linearity eliminates the possibility to superimpose the solutions for different excitations. The parameter extraction $\left(R_{t h z}, a_{i}, q_{k}, R_{i, k}\right)$ requires at least 6 simulations or measurements for different values of dissipated power, to obtain the number of equations equal to or greater than the number of parameters. On the contrary, instead of defining 6 boundary temperature values for the linear isothermal boundary condition, only the ambient temperature must be given for the non-linear model with convection. The only input variables for the whole model are $P$, the power dissipated in the junction and $T_{\text {ot }}$, the ambient temperature. The values of junction temperature $T_{z}$ and boundary temperature $T_{i}$ are obtained from the simulations.

\section{Linear model verification}

The linear model was verified for a simple structure of 2-d bar with isolated top and bottom surfaces (Figure 2). The heat was generated in the middle of the bar and flew out via the side surfaces. This structure can be modelled by a 2-node compact model with the number of parameters $\left(R_{t h z}, a_{i}, q_{k}, R_{i, k}\right)$ equal to 9 .

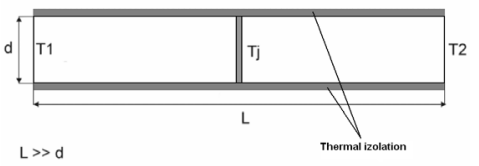

$2 a$

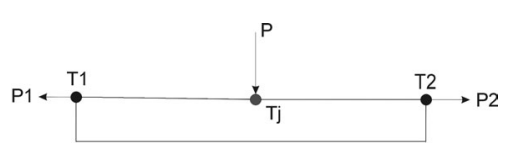

$2 b$

Fig. 2. 2-node linear model

Three ANSYS simulations, each for a different non-zero excitation $\left(P, T_{1}, T_{2}\right)$ give three different sets of output data (Table 1) needed for the parameter extraction (Table 2).

Table 1. Parameter extraction input data, obtained from simulation

\begin{tabular}{|l|l|l|l|}
\hline & $\begin{array}{l}\mathrm{P}=1000, \mathrm{~T}_{1}=273,15 \\
\mathrm{~T}_{2}=273,15\end{array}$ & $\mathrm{P}=0, \mathrm{~T}_{1}=300, \mathrm{~T}_{2}=273,15$ & $\mathrm{P}=0, \mathrm{~T}_{2}=300, \mathrm{~T}_{1}=273,15$ \\
\hline $\mathrm{T}_{\mathrm{z}}[\mathrm{K}]$ & 329,8 & 285,08 & 285,08 \\
\hline $\mathrm{P}_{1}[\mathrm{~W}]$ & 500 & $-118,4$ & 118,4 \\
\hline $\mathrm{P}_{2}[\mathrm{~W}]$ & 500 & 118,4 & $-118,4$ \\
\hline
\end{tabular}


Table 2. Obtained compact model parameters

\begin{tabular}{|l|l|l|l|}
\hline$R_{\text {thz }}$ & 0,05 & $R_{11}$ & $-2,5$ \\
\hline$a_{1}$ & 0,49 & $R_{12}$ & 2,5 \\
\hline$a_{2}$ & 0,49 & $R_{21}$ & 2,5 \\
\hline$q_{1}$ & 0,5 & $R_{22}$ & $-2,5$ \\
\hline$q_{2}$ & 0,5 & & \\
\hline
\end{tabular}

The obtained linear compact model was then validated by comparison with the finite element ANSYS model. The junction temperature calculated using the compact model were compared to the corresponding ANSYS simulation output values (Table 3 , Figure 3 ).

Table 3. Junction temperature obtained

$\mathrm{T}_{\mathrm{z} 1}$ - from the linear compact model, $\mathrm{T}_{\mathrm{z} 2}-$ from the ANSYS simulation

\begin{tabular}{|l|l|l|l|l|l|l|l|l|l|l|}
\hline $\mathrm{P}[\mathrm{W}]$ & 100 & 200 & 300 & 400 & 500 & 600 & 700 & 800 & 900 & 1000 \\
\hline Tz1 [K] & 304,2 & 310 & 315,87 & 321,68 & 327,4 & 333,3 & 339,1 & 344,9 & 350,7 & 356,5 \\
\hline Tz2 $[\mathrm{K}]$ & 305,6 & 311,38 & 317 & 322,67 & 328,3 & 334 & 339,6 & 345,3 & 351 & 356,6 \\
\hline
\end{tabular}

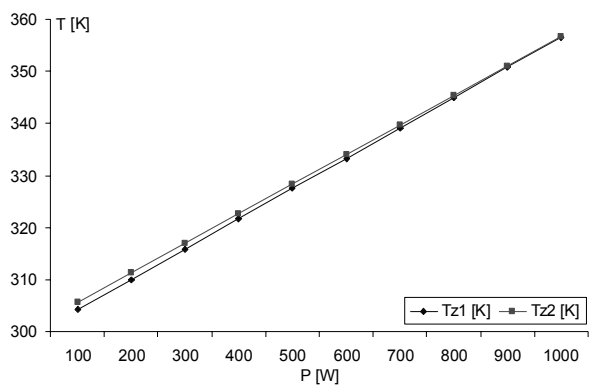

Fig. 3. Comparison of the junction temperature from the linear compact model and from the ANSYS simulation.

\section{Comaprison and verification of the compact models}

In order to compare the basic linear compact model and the new non-linear compact model with convection, their parameters were derived according to the above descriptions (pt 3 and 4). As a reference for both models a series of ANSYS simulations was performed for the dissipated power ranging from 3 to 20 [W]. The junction temperature values obtained for different values of dissipated power are presented below (Table 4, Figure 4).

Table 4. Junction temperature for the linear compact model and the non-linear compact model with convection

\begin{tabular}{|l|l|l|l|l|l|l|l|l|l|}
\hline $\mathrm{P}[\mathrm{W}]$ & 3,53 & 4,82 & 7,29 & 11,2 & 14,83 & 17,04 & 19,13 & 19,59 \\
\hline \multirow{2}{|l|}{ linear model } & \multirow{2}{*}{$\mathrm{T}[\mathrm{K}]$} & 325,1 & 334,5 & 350,6 & 379,4 & 401,9 & 416,9 & 431,4 & 434,3 \\
\cline { 3 - 10 } & 327,7 & 340,4 & 354,9 & 365,3 & 373,7 & 378,4 & 382,6 & 383,5 \\
\hline
\end{tabular}




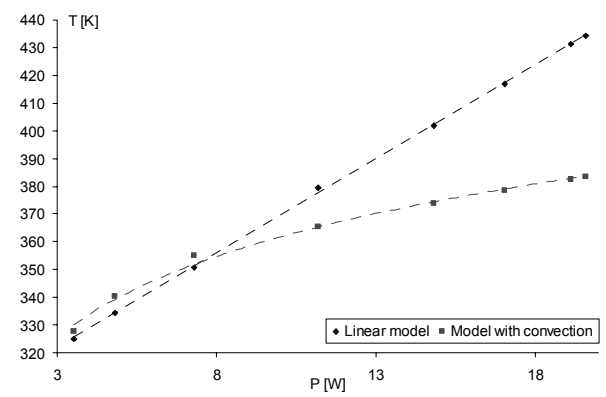

Fig. 4. Comparison of the compact models

Figure 4 shows that the linear model can not fully describe the real phenomena of heat transfer. The non-linear compact model of known parameters $R_{\text {thz }}, a_{i}, q_{k}, R_{i, k}$ permits to calculate the boundary temperatures $T_{i}$ and the junction temperature $T_{z}$ with the dissipated power $P$ and the ambient temperature $T_{\text {ot }}$ being the only input data. The reference thermal measurements and simulations were performed for the power ranging between 3 and 20 [W]. Figure 5 presents the test setup (5a), an example thermal image (5b) and an example ANSYS simulation result (5c). The thermal camera INFRAMETRICS 760 was situated over the examined object with the lens pointing down. The upper face of the object, having the highest temperature, was taken as a reference point. The measured temperature was compared to the results of calculations and simulations (Tables 5 and 6). Figure 6 shows the comparison of the junction temperature $T_{j}(6 a)$ and case temperature $T_{o b}$ (6b) obtained from measurements, simulations and the compact model with convection.

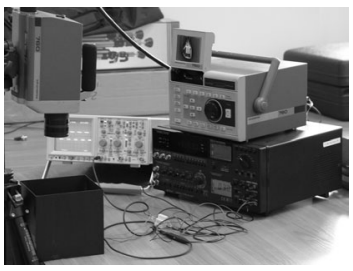

$5 a$

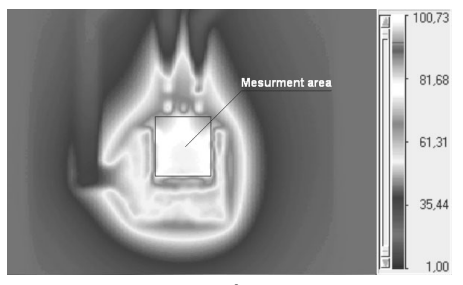

$5 b$

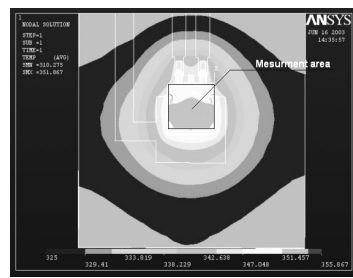

$5 c$

Fig. 5. Test setup, measurement and simulation results.

Table 5. Junction temperature of examined object

\begin{tabular}{|l|l|l|l|l|l|l|l|l|l|}
\hline \multicolumn{2}{|l|}{$\mathrm{P}[\mathrm{W}]$} & 3,53 & 4,82 & 7,29 & 11,2 & 14,83 & 17,04 & 19,13 & 19,59 \\
\hline ANSYS model & \multirow{2}{*}{$\mathrm{T}[\mathrm{K}]$} & 314,98 & 321,26 & 332,73 & 351,54 & 365,25 & 374,34 & 382,8 & 384,71 \\
\cline { 3 - 10 } & 327,72 & 340,42 & 354,98 & 365,39 & 373,76 & 378,42 & 382,61 & 383,51 \\
\hline
\end{tabular}

Table 6. Case temperature of examined object

\begin{tabular}{|c|c|c|c|c|c|c|c|c|c|}
\hline $\mathrm{P}[\mathrm{W}]$ & & 3,53 & 4,82 & 7,29 & 11,2 & 14,83 & 17,04 & 19,13 & 19,59 \\
\hline Measurements & \multirow{3}{*}{$\mathrm{T}[\mathrm{K}]$} & 317,91 & 321,24 & 334,1 & 351,99 & 368,53 & 378,46 & 386,29 & 391,01 \\
\hline Compact model & & 312,74 & 315,6 & 345,24 & 357,02 & 366,24 & 371,31 & 375,81 & 376,76 \\
\hline ANSYS model & & 314,46 & 320,55 & 331,64 & 348,63 & 363,08 & 371,85 & 380,07 & 381,96 \\
\hline
\end{tabular}




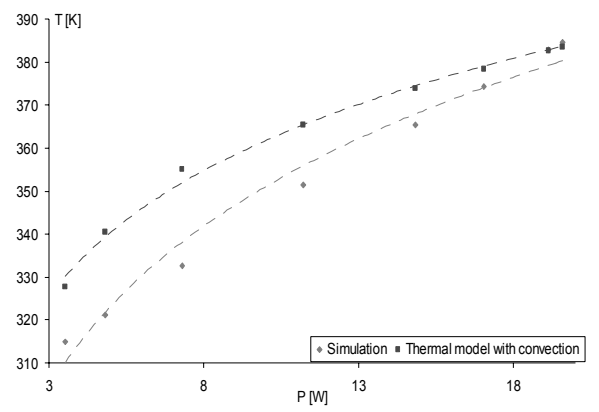

$6 a$

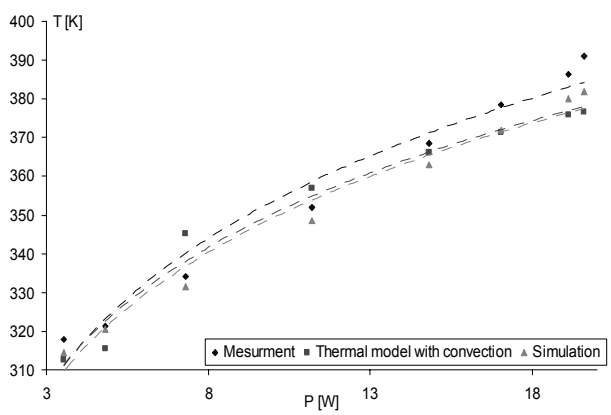

$6 b$

Fig. 6. Comparison of junction temperature (a) and case temperature (b)

\section{Conclusion}

The verification of the compact thermal model confirms heat its parameters are extracted correctly. The junction temperature as a function of power (Figure 6a) according to the compact model with convection shows a good agreement with the simulation results. The divergence of the results is within the acceptable numerical or approximation error level. The surface (case) temperature thermal camera measurements and the values from the compact model are comparable as well.

The results from the linear model compared to the non-linear model with convection (Figure 4) show that the isothermal boundary condition does not fit the reality properly and is not suitable neither for the parameter extraction, nor for the calculation of the junction temperature.

The introduction of convection as a non-linear boundary condition simplifies the compact model parameter extraction and allows correct calculation of the junction temperature.

The compact thermal model described by (10) and (11) can be used for calculation of thermal parameters of electronic components and the maximum allowable junction temperature.

\section{REFERENCES}

[1] TALAR J J., DUDA P. - Rozwiazywanie prostych i odwrotnych zagadnień przewodzenia ciepła, WNT, Warszawa 2003.

[2] GERSTENMAIER Y. C., PAPE H. and WACHUTKA G. - Rigorus Model and Network for Static Thermal Problems. 7th THERMINIC Workshop, 24-27 September 2001, Paris, str. 203-208.

[3] DULNIEW G.N. - Wymiana ciepła w urządzeniach elektronicznych i ich elementach, WNT, Warszawa 1967

[4] WIŚNIEWSKI S. - Wymiana ciepła, PWN, Warszawa 1979.

[5] LIS M., WIĘCEK B., WAJMAN T. - "hermography validation of thermal compact modelling of power electronic components, QIRT'02, Dubrownik, Croatia.

[6] LIS M., ROSOWSKI A. - Thermal Simulation of Power MOS Transistor in D2Pack Case with Use of ANSYS Software, 5th International Seminar MicroTherm'2003, Łódź. 
http://dx.doi.org/10.21611/qirt.2004.072 